\title{
Author Index Volume 16
}

H. Abdel-Jaber Experimental Analysis of Students'

Satisfaction Factors in E-Learning Environment: A Case

Study on Saudi Arabian University

2 (2017) 1750018

I. S. Abdullah Influences of Knowledge Sharing on Individual Performance in Saudi Organisations

2 (2017) 1750019

K. Ahmad The Perspective of Library and Information

Science (LIS) Professionals Toward Knowledge

Management in University Libraries

2 (2017) 1750015

E. M. Ahmed \& T. K. Yang High Attrition Rate Determinants: Case Study of the Malaysian ICT Sector

3 (2017) 1750030

3 (2017) 1750028

3 (2017) 1750023

R. Alhajj see Suleiman, I.

1 (2017) 1750002

1 (2017) 1750002

R. Almestarihi see Migdadi, M. M.

H. Al-Mofareji, M. Kamel \& M. Y. Dahab WeDoCWT: A New Method for Web Document Clustering Using Discrete Wavelet Transforms

1 (2017) 1750004

D. Alsmadi, C. Jiangping, V. Prybutok \& G. Gadgil ELearning in Jordanian Higher Education: Cultural Perspectives and Institutional Readiness

$4(2017) 1750035$

S. Amin \& S. Aslam Intellectual Capital, Innovation and Firm Performance of Pharmaceuticals: A Study of the London Stock Exchange

2 (2017) 1750017

M. A. Anwar see Rafique, G. M.

4 (2017) 1750041

N. Armaghan \& J. Renaud Evaluation of Knowledge Management in an Organisation

1 (2017) 1750006

M. Arslan see Suleiman, I.

3 (2017) 1750023

2 (2017) 1750017

M. Atapattu \& G. Ranawake Transformational and

Transactional Leadership Behaviours and their Effect on Knowledge Workers' Propensity for Knowledge Management Processes 
U. Ayub, A. R. Kausar \& M. M. Qadri Linking Human Capital and Organisational Innovative Capabilities of Financial Institutions: Evidence from a Developing Country of South Asia

R. Baker see Turner, J. R.

D. Basten, L. Schneider \& O. Pankratz Codification, Personalisation, or in Between? Exploring Knowledge Characteristics to Guide Knowledge Management System Design

P. J. Bateman see Cho, K.

K. Benali \& S. A. Rahal OntoDTA: Ontology-Guided Decision Tree Assistance

I. Boyko see Panikarova, S.

K. A. Bugyei, K. Obiri-Yeboah \& R. K. Kavi Assessing the Impact of ICT on Research Activities in Ghana: A Case Study of Selected Council for Scientific and Industrial Research (CSIR) Institutes

K. Cho, S. Han, T.-T. (R.) Chung \& P. J. Bateman The Influence of an Integrated View of Source's Expertise on Knowledge Transfer

T.-T. (R.) Chung see Cho, K.

A. Coners see Matthies, B.

M. Y. Dahab see Al-Mofareji, H.

C. Dennehy see Uddin, Md. N.

A. El-Halees \& A. Al-Asmar Ontology Based Arabic Opinion Mining

N. F.-H. Farahmand Competition Trading and Exporting Acting by Inter Marketing Managers

A. M. Fauzi see Nur, R. N. N.

M. C. Fombad \& O. B. Onyancha Knowledge Management for Development: Rethinking the Trends of Knowledge Management Research in South Africa

G. Gadgil see Alsmadi, D.

A. Ghazali, A. Tretiakov, D. Pringgabayu \& D. M. A. Ramdlany Analyzing Knowledge Construction in Online Health Group Discussion

W. Hadi A New Model for Integrating Phases of DecisionMaking and Knowledge Base for Improving Customer Satisfaction

S. Han see Cho, K.

P. C. Izunwanne Developing an Understanding of Organisational Knowledge Creation: A Review Framework
4 (2017) 1750042

2 (2017) 1750012

4 (2017) 1750037

4 (2017) 1750033

3 (2017) 1750031

2 (2017) 1750016

1 (2017) 1750007

4 (2017) 1750033

4 (2017) 1750033

4 (2017) 1750038

1 (2017) 1750004

1 (2017) 1750003

3 (2017) 1750028

3 (2017) 1750029

4 (2017) 1750032

3 (2017) 1750021

4 (2017) 1750035

1 (2017) 1750009

3 (2017) 1750025

4 (2017) 1750033

2 (2017) 1750020 
C. Jiangping see Alsmadi, D.

F. Kamalov see Thabtah, F.

M. Kamel see Al-Mofareji, H.

A. R. Kausar see Ayub, U.

R. K. Kavi see Bugyei, K. A.

Md. H.-O.-R. Khandaker see Uddin, Md. N.

R. Kumar \& R. C. Tripathi An Analysis of the Impact of Introducing the Plagiarism Detection System in an Institute of Higher Education

P. P. Kundapur \& L. L. R. Rodrigues Analysis of a Theoretical KMS Model Implementation in the Indian IT Sector Using $P L S-S E M$

W.-H. Lai see Ly, P. T. M.

B. Lak \& J. Rezaeenour Effective Factors of Social Customer Knowledge Management (SCKM) in Organisations: Study of Electronic Service Providers in Iran

P. T. M. Ly \& W.-H. Lai Fuzzy AHP Analysis of Firm-Level Knowledge Accumulation

M. A. Mamun see Uddin, Md. N.

S. M. Mannan see Uddin, Md. N.

B. Matthies \& A. Coners Document Selection for Knowledge Discovery in Texts: Framework Development and Demonstration

M. Mehrotra see Rastogi, A.

M. M. Migdadi, M. K. A. Zaid, M. Yousif, R. Almestarihi \& K. Al-Hyari An Empirical Examination of Knowledge Management Processes and Market Orientation, Innovation Capability, and Organisational Performance: Insights from Jordan

M. S. Mirian see Moradi, R.

S. Misra see Singh, V. B.

R. Moradi, K. Taheri \& M. S. Mirian Data-Driven Methods to Create Knowledge Maps for Decision Making in Academic Contexts

V. A. Nguyen The Impact of Online Learning Activities on Student Learning Outcome in Blended Learning Course

R. N. N. Nur, A. M. Fauzi \& H. Sukoco Strategies of Knowledge Management Implementation for Academic Services Improvement of Indonesian Higher Education

K. Obiri-Yeboah see Bugyei, K. A.

J. O'Brien see Walsh, J. N.

O. B. Onyancha see Fombad, M. C.
4 (2017) 1750035

4 (2017) 1750034

1 (2017) 1750004

4 (2017) 1750042

1 (2017) 1750007

1 (2017) 1750003

$2(2017) 1750011$

1 (2017) 1750001

3 (2017) 1750024

2 (2017) 1750014

3 (2017) 1750024

1 (2017) 1750003

1 (2017) 1750003

4 (2017) 1750038

4 (2017) 1750036

1 (2017) 1750002

1 (2017) 1750008

1 (2017) 1750005

1 (2017) 1750008

4 (2017) 1750040

4 (2017) 1750032

1 (2017) 1750007

4 (2017) 1750039

3 (2017) 1750021 
S. Panikarova, M. Vlasov \& I. Boyko Assessing Research Productivity in University Environment: Institutional Approach

2 (2017) 1750016

O. Pankratz see Basten, D.

4 (2017) 1750037

M. Patwardhan see Sekhar, C.

3 (2017) 1750022

D. Pringgabayu see Ghazali, A.

1 (2017) 1750009

V. Prybutok see Alsmadi, D.

4 (2017) 1750035

M. M. Qadri see Ayub, U.

4 (2017) 1750042

N. Rabiee Study on Barriers to Knowledge Management Implementation in Iranian Companies; Case Study: PDSCO (Projects Development and Solution Company)

$2(2017) 1750013$

G. M. Rafique \& M. A. Anwar Motivating Knowledge Sharing Among Undergraduate Medical Students of the University of Lahore, Pakistan

S. A. Rahal see Benali, K.

Md. S. Rahman see Uddin, Md. N.

D. M. A. Ramdlany see Ghazali, A.

G. Ranawake see Atapattu, M.

A. Rastogi \& M. Mehrotra Opinion Spam Detection in Online Reviews

J. Renaud see Armaghan, N.

J. Rezaeenour see Lak, B.

M. Ridley see Suleiman, I.

L. L. R. Rodrigues see Kundapur, P. P.

J. Sack see Uddin, Md. N.

L. Schneider see Basten, D.

C. Sekhar, M. Patwardhan \& V. Vyas Study of Intellectual Capital Dimensions in IT Industry Using DEMATEL Method

3 (2017) 1750022

1 (2017) 1750005

V. B. Singh, S. Misra \& M. Sharma Bug Severity Assessment in Cross Project Context and Identifying

Training Candidates

4 (2017) 1750041

3 (2017) 1750031

1 (2017) 1750003

1 (2017) 1750009

3 (2017) 1750026

4 (2017) 1750036

1 (2017) 1750006

2 (2017) 1750014

3 (2017) 1750023

1 (2017) 1750001

1 (2017) 1750003

4 (2017) 1750037

H. Sukoco see Nur, R. N. N.

1 (2017) 1750005

4 (2017) 1750032

I. Suleiman, M. Arslan, R. Alhajj \& M. Ridley Prediction Model of School Readiness

3 (2017) 1750023

1 (2017) 1750008

K. Taheri see Moradi, R.

F. Thabtah \& F. Kamalov Phishing Detection: a Case Analysis on Classifiers with Rules Using Machine Learning

4 (2017) 1750034

A. Tretiakov see Ghazali, A.

1 (2017) 1750009

R. C. Tripathi see Kumar, R.

2 (2017) 1750011 
J. R. Turner \& R. Baker Team Emergence Leadership Development and Evaluation: a Theoretical Model Using Complexity Theory

$2(2017) 1750012$

Md. N. Uddin, Md. S. Rahman, Md. H.-O.-R. Khandaker, M. A. Mamun, S. M. Mannan, J. Sack \& C. Dennehy Use and Impact of HINARI: An Observation in Bangladesh with Special Reference to $I c d d r, b$

1 (2017) 1750003

M. Vlasov see Panikarova, S.

2 (2017) 1750016

V. Vyas see Sekhar, C.

3 (2017) 1750022

J. N. Walsh \& J. O'Brien A Knowledge-Based Framework for Service Management

4 (2017) 1750039

T. K. Yang see Ahmed, E. M.

3 (2017) 1750030

D. K. Yoo Impacts of a Knowledge Sharing Climate and Interdisciplinary Knowledge Integration on Innovation

2 (2017) 1750010

M. Yousif see Migdadi, M. M.

1 (2017) 1750002

M. K. A. Zaid see Migdadi, M. M.

1 (2017) 1750002 